\title{
Prevalência e fatores associados ao doping cosmético entre frequentadores de academias
}

\section{Prevalence and associated factors to the cosmetic doping among fitness-centers customers}

\author{
Jhonatan Lima-Oliveira ${ }^{1}$, Paulo Adriano Schwingel ${ }^{1,2}$ \\ 'Departamento de Nutrição, Universidade de Pernambuco (UPE) - Petrolina (PE), Brasil. \\ ${ }^{2}$ Faculdade de Medicina da Bahia (FMB), Universidade Federal da Bahia (UFBA) - Salvador (BA), Brasil.
}

DOI: http://dx.doi.org/10.7322/abcshs.v40i1.704

\section{RESUMO}

Introdução: O crescente culto ao corpo na sociedade atual tem levado diversos frequentadores de academia à prática sistemática de injetar óleos por via intramuscular, procedimento denominado doping cosmético. Assim, o presente estudo teve como objetivo verificar a prevalência de doping cosmético na região do Vale do São Francisco, buscando estabelecer os perfis sociodemográfico e clínico desse grupo de indivíduos. Relato de caso: Trata-se de um estudo retrospectivo e documental, com análise dos registros de 346 frequentadores de academias de ginástica, dos quais sete relataram utilização de doping cosmético. Destes registros, foram coletados dados sociodemográficos, cineantropométricos, hemodinâmicos, história patológica pessoal e familiar pregressa e de consumo de drogas lícitas e ilícitas. A prevalência de doping cosmético foi 2,0\% (IC95\% 0,8-4,1), e a maioria dos usuários são adultos jovens com segundo grau completo, trabalhadores de classe média, sendo considerados ativos com baixo percentual de gordura. Eles utilizam suplementos alimentares, esteroides anabolizantes e outras drogas ilícitas. Conclusão: Os achados contribuem na determinação do perfil dessa população, demonstrando que os usuários de doping cosmético são adultos jovens preocupados com a estética corporal. As características sociodemográficas encontradas diferem de estudos prévios quanto ao nível de escolaridade, renda e ocupação. Nesse sentido, ações voltadas para a conscientização dessa população quanto aos riscos e perigos da utilização do doping cosmético devem ser apoiadas o mais breve possível.

Palavras-chave: doping nos esportes; injeções intramusculares; óleo mineral; óleos vegetais; fatores de risco.

\begin{abstract}
Introduction: The rise in the cult of the body in modern society has led many fitness-centers customers to the systematic practice of intramuscular oil injections, procedure called cosmetic doping. Therefore, this study aimed to determine the prevalence of cosmetic doping in the São Francisco Valley region, in order to establish the sociodemographic and clinical profiles of this group of individuals. Case report: This is a retrospective documentary study with analysis of 346 records from fitness-centers customers, of which 7 reported use of cosmetic doping. Sociodemographic, kinanthropometric, hemodynamic, personal and family past medical history and the consumption of licit and illicit drug data were collected from these records. The prevalence of cosmetic doping was $2.0 \%$ (95\% Cl 0.8-4.1), and most of the users are young adults with high school graduates, middle-class workers, and are considered physically active with low body fat mass. They use concomitantly dietary supplements, anabolic steroids and other illicit drug. Conclusions: The findings of the study contribute in determining the profile of this population, demonstrating that users of cosmetic doping are young adults concerned with aesthetic body. The sociodemographic characteristics found in this study differ from previous studies in the level of education, income and occupation. In this sense, actions to raise awareness of this population about the risks and dangers of the cosmetic doping should be supported as soon as possible.
\end{abstract}

Keywords: doping in sports; injections, intramuscular; mineral oil; plant oils; risk factors. 


\section{INTRODUÇÃO}

A preocupação do homem com a imagem corporal não é recente. Atualmente, o culto ao corpo vem se desenvolvendo e ganhando adeptos por todo o mundo ${ }^{1}$. No Brasil, tal preocupação se reflete também no crescente número de usuários de esteroides anabolizantes androgênicos (EAA) ${ }^{2}$.

Nesse sentido, os olhares se voltaram predominantemente a um padrão estético corporal desejável na visão moderna. Ou seja, indivíduo saudável não é mais aquele que apresenta uma aparência nutrida, mas sim o que apresenta imagem corporal hipermusculosa ${ }^{3}$. Além disso, a dieta habitual utilizada por esse perfil de indivíduo não visa apenas suprir necessidades energéticas, mas "turbinar o corpo", sendo principalmente hiperproteica e hipolipídica, visando manter uma boa aparência, muitas vezes considerada midiática ${ }^{1,2}$.

Com esse intuito, a busca por um corpo perfeito tem levado diversos praticantes de atividades físicas a utilizar substâncias e/ou compostos que aprimoram o desempenho físico, sejam eles lícitos ou não, fato que pode comprometer seriamente a sua saúde ${ }^{3}$. Um procedimento em ascensão tem sido a aplicação intramuscular de óleos, como o polivitamínico ADE. Esse procedimento tem sido denominado doping cosmético ${ }^{4}$ e vem ganhando a simpatia de diversos frequentadores de academias de ginástica e/ou musculação, com a finalidade de competição ou mesmo para realização pessoal, por trazer resultados considerados extremamente rápidos.

Doping cosmético é a prática sistemática de injetar por via intramuscular substâncias oleosas, como os óleos minerais e o complexo polivitamínico denominado ADE (mistura das vitaminas lipossolúveis $\mathrm{A}, \mathrm{D}$ e $\mathrm{E}$ ), que geram aumento volumétrico localizado. Esse aumento, no entanto, não é relacionado à hipertrofia muscular ou a qualquer evento adaptativo fisiológico, tendo apenas efeito cosmético e resultando na deformidade da aparência original do músculo. O volume injetado, que é, muitas vezes, superior à dosagem dada a animais, propósito original do ADE, ocupa espaço no local de aplicação, dentro do ventre muscular ou no tecido subcutâneo adjacente ${ }^{4}$. Além desse tradicional polivitamínico, na literatura há relatos de injeções intramusculares de óleos de gergelim ${ }^{5}$ e de parafina ${ }^{6}$ por praticantes de musculação e/ou fisiculturismo. Ressalta-se, porém, que os efeitos adversos verificados podem variar desde inflamações agudas até necrose $e^{5,6}$, com possível amputação da região comprometida.

Contrariamente à crescente expansão no número de estudos que avaliam o uso de $\mathrm{EAA}^{1}$, os que avaliam indivíduos que fazem uso de doping cosmético são escassos ${ }^{4}$, demonstrando ser esta uma temática negligenciada em âmbito nacional. Somado a isso, há a crescente preocupação quanto ao número cada vez maior de praticantes de atividades físicas que se utilizam dessa prática. Estudo recente ${ }^{7}$ relata que apesar de o doping cosmético apresentar problemas, estes podem levar até décadas para aparecer, o que pode interferir na baixa procura médica por parte dos usuários desse método.
Portanto, o presente estudo teve como objetivo verificar a prevalência de doping cosmético entre frequentadores de academias de ginástica da região integrada de desenvolvimento (RIDE) do polo Petrolina, em Pernambuco, e Juazeiro, na Bahia, buscando estabelecer perfis sociodemográfico e clínico desse grupo de indivíduos.

\section{RELATO DE CASO}

Este trabalho é um relato de caso retrospectivo, elaborado por meio da revisão de sete registros de frequentadores de academias de ginástica da RIDE do polo Petrolina-Pernambuco e JuazeiroBahia, que foram avaliados entre março e novembro de 2012 no Laboratório de Avaliação Nutricional da Universidade de Pernambuco, Campus Petrolina.

Foram incluídos praticantes de musculação regularmente matriculados em academias registradas na RIDE do polo Petrolina-Pernambuco e Juazeiro-Bahia que entregaram Termo de Consentimento Livre e Esclarecido (TCLE) devidamente datado e assinado e que reportaram utilizar doping cosmético. Indivíduos com menos de 18 anos de idade entregaram o TCLE devidamente datado e assinado pelo pai ou responsável. Voluntários que apresentaram dificuldades motoras, distúrbios articulatórios ou alteração no estado físico, comportamental e/ou psicológico que impossibilitassem a realização dos testes ou o preenchimento dos instrumentos de coleta foram excluídos.

Das fichas clínicas de avaliação e das entrevistas face a face, foram retirados dados quali-quantitativos sociodemográficos (sexo, idade, escolaridade, ocupação, renda familiar), cineantropométricos (massa corporal total, estatura, índice de massa corporal e percentual de gordura), hemodinâmicos (pressão arterial e frequência cardíaca), clínicos (história patológica pregressa e antecedentes familiares) e de consumo de drogas lícitas e ilícitas (etilismo, tabagismo, uso de suplementos dietéticos, consumo de narcóticos, uso de EAA, percepção de efeitos colaterais, local de obtenção dos compostos, tempo de utilização, número de injeções intramusculares, local das aplicações, etc.).

As avaliações antropométricas seguiram a padronização da International Society for the Advancement of Kinanthropometry $(\text { ISAK })^{8}$. O percentual de gordura registrado na avaliação nutricional foi obtido a partir da análise de bioimpedância (BIA) com auxílio do aparelho bipolar portátil HBF-306C (Omron Healthcare Inc., Lake Forest, IL, Estados Unidos) seguindo a padronização inerente ao teste ${ }^{9}$. Obteve-se o índice de massa corporal (IMC) dividindo o valor da massa corporal total em quilogramas pelo valor da estatura ao quadrado, em metros $\left(\mathrm{kg} / \mathrm{m}^{2}\right)$. O nível de atividade física foi avaliado pelo questionário internacional de atividade física (iPAQ) versão curta de forma autoaplicável ${ }^{10}$.

Para a mensuração dos valores de pressão arterial (PA), utilizou-se o método oscilométrico por meio do monitor de PA de braço automático Omron HEM-742INT (Omron Healthcare Inc., Lake Forest, IL, Estados Unidos). Utilizaram-se manguitos distintos de 
acordo com a circunferência do braço do avaliado (H-CR17: 22 a $32 \mathrm{~cm}$; HEM-CL24: 32 a $42 \mathrm{~cm}$ ). Todas as avaliações da medida da PA foram realizadas no braço direito de cada indivíduo, que estava sentado, após 10 minutos de repouso.

Os dados coletados analisados estão apresentados em valores individuais. O presente estudo foi aprovado pelo Comitê de Ética em Pesquisa com seres humanos da Maternidade Climério de Oliveira da Universidade Federal da Bahia (Parecer/Resolução $n^{\circ}$ 04/2007). Para preservar o anonimato dos participantes, os nomes dos voluntários foram trocados por códigos numéricos individuais.

Das 346 fichas de entrevista coletadas, verificou-se uso de substâncias consideradas como doping cosmético por sete $(2,0 \%)$ indivíduos (intervalo de confiança [IC] de 95\% 0,8-4,1), sendo todos do sexo masculino. Seis $(85,7 \%)$ entrevistados relataram injetar ADE, enquanto um (14,3\%) reportou injeções de ADE e óleo mineral. A maioria $(85,7 \%)$ estava inscrito na academia de ginástica reportada há pelo menos dois anos. Todos $(100,0 \%)$ foram considerados ativos de acordo com o iPAQ, sendo $2(28,6 \%)$ moderadamente ativos e $5(71,4 \%)$ muito ativos. A mediana (primeiro quartil - terceiro quartil) de idade foi 21,0 $(19,5-30,0)$ anos. A maioria $(85,7 \%)$ dos usuários de doping cosmético tinha concluído o segundo grau, e um destes possuía nível superior completo. Cinco $(71,4 \%)$ indivíduos relataram renda familiar entre 3 e 5 salários-mínimos e 1 (14,3\%) reportou renda superior a 5 salários (Tabela 1 ).

A massa corporal total dos usuários variou entre 68,8 e 112,9 kg e a estatura entre 167,0 e 194,0 cm. De acordo com a categorização do IMC, três indivíduos apresentaram excesso de peso corporal (um com sobrepeso e dois com obesidade). Entretanto, de acordo com o percentual de gordura obtido pela BIA, apenas o indivíduo com registro de número 11 pode ser considerado com excesso de peso, sendo classificado como obeso grau I (Tabela 2).
Ressalta-se, porém, que os indivíduos registrados com os números 16 e 20 apresentaram baixo percentual de gordura para suas respectivas faixas etária. Já em relação à pressão arterial sistólica (PAS) e à diastólica (PAD), verificou-se que os indivíduos 11 e 246 apresentaram alteração pontual na PAS.

Apesar de normalmente os frequentadores de academia absterem-se do consumo de álcool e drogas ilícitas, chamam a atenção os dados relacionados ao uso desses compostos de forma concomitante com o doping cosmético. Cinco $(71,4 \%)$ indivíduos faziam uso de bebidas alcoólicas entre uma e duas vezes na semana, enquanto dois $(28,6 \%)$ relataram tabagismo. Em relação ao uso de drogas ilícitas, $71,4 \%$ relatou já ter utilizado, e um (14,3\%) indivíduo reportou consumir regularmente a droga conhecida como "loló" (fórmula composta por benzina, clorofórmio, éter e essência perfumada). Essa mesma droga também foi reportada por todos os quatro ex-usuários de drogas. Três indivíduos também confirmaram uso pregresso de maconha, e um avaliado reportou consumo prévio de crack. Um indivíduo que havia reportado consumos prévios de "loló" e maconha também confirmou utilizar ecstasy em festas. Quanto a efeitos colaterais reportados, dois $(28,5 \%)$ indivíduos relataram agressividade ou alterações de humor, e outros dois $(28,5 \%)$ reportaram cefaleia e/ou dor de cabeça difusa (Tabela 3).

Com relação à aquisição dos compostos para aumento de volume muscular, a maioria dos indivíduos relatou adquirir em farmácias ou lojas veterinárias sem apresentação de receitas. Seis $(85,7 \%)$ afirmaram ter sido um amigo quem aplicou o composto em domicílio sem acompanhamento de profissional da área da saúde que soubesse o correto manuseio dos equipamentos de injeção. Apenas um indivíduo afirmou ter sido o farmacêutico quem aplicou o composto. Nenhum dos indivíduos teve acompanhamento médico ou de outro profissional especializado durante o tempo de uso desses compostos, e todos relataram não

Tabela 1: Características sociodemográficas dos sujeitos

\begin{tabular}{|l|l|c|c|c|c|}
\hline Indivíduo & Sexo & Idade (anos) & Escolaridade & Ocupação & Renda familiar (salários mínimos) \\
\hline 11 & Masculino & 21 & Ensino Médio & Autônomo & Mais de 5 \\
\hline 12 & Masculino & 31 & Ensino Fundamental & Recepcionista & Entre 3 e 5 \\
\hline 16 & Masculino & 29 & Ensino Superior & Autônomo & Entre 3 e 5 \\
\hline 20 & Masculino & 37 & Ensino Médio & Funcionário público & Entre 3 e 5 \\
\hline 31 & Masculino & 20 & Ensino Médio & Pintor & Entre 1 e 2 \\
\hline 246 & Masculino & 19 & Ensino Médio & Vendedor & Entre 3 e 5 \\
\hline 270 & Masculino & 16 & Ensino Médio & Estudante & Entre 3 e 5 \\
\hline
\end{tabular}

Tabela 2: Características cineantropométricas e hemodinâmicas dos sujeitos

\begin{tabular}{|c|c|c|c|c|c|c|c|}
\hline Indivíduo & Estatura (cm) & Peso (kg) & IMC $\left(\mathrm{kg} / \mathrm{m}^{2}\right)$ & Gordura (\%) & PAS $(\mathrm{mmHg})$ & PAD (mmHg) & FC repouso (bpm) \\
\hline 11 & 194,0 & 112,9 & 30,0 & 28,8 & 130 & 80 & 112 \\
\hline 12 & 177,0 & 74,3 & 23,7 & 19,8 & 108 & 68 & 75 \\
\hline 16 & 171,0 & 72,5 & 24,8 & 14,2 & 123 & 76 & 49 \\
\hline 20 & 172,0 & 88,9 & 30,1 & 18,8 & 119 & 61 & 82 \\
\hline 31 & 167,0 & 75,1 & 26,9 & 14,0 & 120 & 78 & 67 \\
\hline 246 & 182,0 & 75,5 & 22,8 & 13,1 & 139 & 68 & 100 \\
\hline 270 & 178,0 & 68,8 & 21,7 & 11,7 & 127 & 56 & 76 \\
\hline
\end{tabular}

IMC: índice de massa corporal; PAS: pressão arterial sistólica; PAD: pressão arterial diastólica; FC: frequência cardíaca 
utilizar drogas que melhoravam o desempenho físico no momento das consultas (Tabela 4).

Os registros da entrevista face a face permitiram verificar que todos os indivíduos utilizaram doping cosmético para estética corporal e definição muscular. Os entrevistados que utilizaram EAA de forma concomitante informaram que recorriam a esse procedimento visando ganho de força e aumento da área de seção transversa muscular. Ainda, os dados clínicos revelaram que os indivíduos 11 e 20 reportavam epilepsia e bipolaridade, respectivamente. Por fim, quatro $(57,1 \%)$ indivíduos apresentavam histórico familiar de hipertensão arterial sistêmica e estavam inseridos em grupo de risco para doenças cardiovasculares.

\section{DISCUSSÃO}

O principal achado deste estudo ressalta a crescente procura pela estética e perfeição corporal por frequentadores de academias de ginástica, uma vez que o doping cosmético foi prevalente em $2 \%$ desses indivíduos. Apesar de a aplicação intramuscular de óleos no presente estudo ter sido verificada apenas em registros de indivíduos do sexo masculino, na literatura esse procedimento não está restrito a esse gênero ${ }^{3,4,7}$.

Embora o doping cosmético comece a figurar como possível problema de saúde pública, verifica-se que não existem dados epidemiológicos suficientes sobre esse tema, sendo a maioria das publicações relatos de caso $^{7,11}$. Figueiredo et al. ${ }^{4}$ chamavam a atenção já em 2011 para a escassez de pesquisas que abordam o presente tema. Dos poucos trabalhos que abordam doping cosmético, Iriart e Andrade ${ }^{12}$ e Schwingel e Cotrim ${ }^{13}$ observaram esta prática no mesmo município (Salvador, Bahia), porém em estudos de características socioantro- pológicas conduzidos com praticantes de musculação e/ou fisiculturismo que consumiam EAA. Apenas um estudo de caráter exploratório conduzido em João Pessoa, Paraíba ${ }^{14}$, investigou praticantes de musculação em academias de ginástica e verificou prevalência de doping cosmético de 11,0\% (IC95\% $8,4-14,0)$. Nesse sentido, o presente estudo torna-se inovador ao analisar o doping cosmético entre frequentadores de academia de ginástica de uma região sertaneja e de características distintas das grandes metrópoles brasileiras. Apesar de a prevalência verificada no presente estudo $(2,0 \%)$ ter sido inferior à reportada em estudos prévios ${ }^{13,14}$, esse resultado demonstra que a prática de doping cosmético não está restrita às regiões metropolitanas brasileiras e pode ser interpretado como preocupante, uma vez que, embora baixa, não é nula.

É válido notar que a maioria dos indivíduos que utilizaram esses métodos rápidos de construção corporal no presente estudo não está encaixada no que seriam os estereótipos comumente apresentados na literatura: fisiculturistas amadores e profissionais com o intuito de melhores resultados ${ }^{4}$ ou praticantes de musculação de academias clandestinas com baixas renda e escolaridade ${ }^{12,13}$. Os registros analisados são oriundos de indivíduos com escolaridade média ou superior e renda maior que três salários mínimos. Também preocupa o fato de a maioria dessa amostra não ser composta de atletas amadores ou profissionais, mas de praticantes regulares ou sazonais de musculação.

A problemática, portanto, está em perceber que, se essa população entendida como mais bem informada está a escolher esses meios e/ou caminhos, quais problemas não serão encontrados se os olhares se voltarem aos frequentadores de academias desinformados. Mesmo que a mídia apresente constantes alertas e casos de pessoas que tiveram sérias complicações em decorrência do

Tabela 3: Compostos utilizados concomitantemente ao doping cosmético

\begin{tabular}{|l|c|c|c|c|c|c|}
\hline Indivíduo & Etilismo & Tabagismo & Narcóticos & Uso de suplementos & Uso de EAA & Efeitos colaterais \\
\hline 11 & Sim & Não & Já utilizou & Sim (diversos) & Nunca utilizou & Cefaleia \\
\hline 12 & Sim & Não & Já utilizou & Sim (diversos) & Já utilizou & Cefaleia \\
\hline 16 & Sim & Não & Nunca utilizou & Sim (diversos) & Já utilizou & Nenhum \\
\hline 20 & Ex $^{*}$ & Ex & Utiliza & Sim (diversos) & Já utilizou & Agressividade \\
\hline 31 & Sim & Ex & Nunca utilizou & Sim (diversos) & Já utilizou & Nenhum \\
\hline 246 & Sim & Não & Já utilizou & Sim (diversos) & Já utilizou & Agressividade \\
\hline 270 & Não & Não & Já utilizou & Sim (diversos) & Já utilizou & Nenhum \\
\hline
\end{tabular}

*Reportou ter parado de consumir álcool há pelo menos um ano, sendo considerado ex-etilista. * ${ }^{*}$ Reportou ser ex-tabagista, tendo parado de fumar há pelo menos seis meses. EAA: esteroides anabolizantes androgênicos

Tabela 4: Informações sobre o uso de drogas que melhoram a estética corporal

\begin{tabular}{|l|c|c|c|c|c|c|}
\hline Indivíduo & Obtenção do ADE & Tempo de uso/aplicações & Ambiente de aplicação & Aplicador & Informações \\
\hline 11 & Veterinária & $1 \mathrm{mês} / 2$ vezes & Domicílio & Amigo & Amigo \\
\hline 12 & Amigo & $1 \mathrm{mês} / 2$ vezes & Domicílio & Amigo & Amigo \\
\hline 16 & Amigo & $1 \mathrm{mês} / 1 \mathrm{vez}$ & Academia & Amigo & Amigo \\
\hline 20 & Farmácia & $1 \mathrm{mês} / 1 \mathrm{vez}$ & Farmácia & Farmacêutico & Na academia \\
\hline 31 & Amigo & $1 \mathrm{mês} / 3 \mathrm{vezes}$ & Domicílio & Amigo & Amigo \\
\hline 246 & Farmácia & $1 \mathrm{mês} / 1 \mathrm{vez}$ & Domicílio & Amigo & Amigo e na academia \\
\hline 270 & Farmácia, amigo e veterinária & $1 \mathrm{mês} / 2$ vezes & Domicílio & Amigo & Amigo e na academia \\
\hline
\end{tabular}

ADE: polivitamínico (mistura das vitaminas lipossolúveis A, D e E) 
não acompanhamento durante a utilização de diversos tipos de compostos ${ }^{7}$, casos de utilização de drogas que melhoram a estética corporal são cada vez mais frequentes ${ }^{1-7,12,13}$.

Em relação à aquisição dos compostos, verificou-se elevada prevalência quanto à obtenção por via ilegal ou por meio do denominado mercado negro. Os compostos foram obtidos em farmácias e lojas veterinárias sem apresentação de receita. Além disso, é importante ressaltar que a maior parte dos usuários recebeu a aplicação de um amigo ou conhecido, em ambiente domiciliar e sem instrução de qualquer profissional devidamente habilitado. Nesse aspecto, necrose e graves sequelas musculares têm sido relatadas em decorrência da má aplicação dessa substância ${ }^{4,12}$. Além disso, reações a óleos injetados podem surgir após muitos anos de latência ${ }^{4}$.

Outro ponto que merece destaque foi a elevada frequência de usuários de doping cosmético que consumiam ou já haviam utilizado outras drogas ilícitas de forma concomitante (71,4\%). Apesar de o uso do doping cosmético não apresentar relação com a teoria social da "porta de entrada para o consumo de outras drogas", fato que é comumente ligado ao uso de EAA, o elevado número de usuários de drogas que utilizavam drogas psicotrópicas corrobora com estudos prévios ${ }^{1,15}$. Além disso, os comportamentos de risco à saúde encontrados no presente estudo denotam a necessidade de campanhas de prevenção centradas tanto na desconstrução dos valores associados ao corpo na sociedade atual quanto na veiculação de informação de qualidade sobre os riscos à saúde ocasionados pelo consumo de todas essas drogas ilícitas ${ }^{11}$.

Já em relação aos efeitos colaterais reportados nas avaliações clínicas, verificamos que dois indivíduos relataram cefaleia e dor de cabeça difusa que podem estar associadas à injeção de fármacos em profundidade em um músculo grande. Por sua vez, a agressividade reportada por outros dois indivíduos não pode ser diretamente relacionada ao uso do doping cosmético. Agressividade exacerbada, irritabilidade e agitação motora são efeitos colaterais prevalentes entre usuários ilícitos de testosterona e seus derivados ${ }^{11,12,15}$, estando esses comportamentos possivelmente relacionados à redução na produção de receptores de serotonina em regiões do cérebro relacionadas ao controle da agressividade.

Quanto ao perfil dos usuários de doping cosmético na região estudada, verifica-se que, em sua maioria, são adultos jovens que fazem uso das injeções de forma esporádica ou aguda, considerados ativos e com baixo percentual de gordura. Estes fazem uso de suplementos alimentares e de EAA, têm segundo grau completo, são trabalhadores ou estão empregados. Apesar das características de aptidão física corroborarem estudos anteriores $^{4,11-13}$, o perfil sociodemográfico encontrado difere quanto ao nível de escolaridade, renda e ocupação.

Portanto, o presente estudo traçou o perfil socioeconômico dos usuários de doping cosmético na região estudada, demonstrando que todos eram do sexo masculino, com idade inferior a 40 anos e pertencentes à classe média. Além disso, o uso simultâneo de drogas lícitas e ilícitas foi reportado por todos os sete entrevistados. Diante do exposto e também em razão do crescente número de casos em que usuários de polivitamínicos chegam a óbito, não somente mais estudos sobre o tema são necessários mas, principalmente, ações voltadas para a conscientização dessa população quanto aos riscos e perigos da utilização do doping cosmético, especialmente entre frequentadores de academias de ginástica que busquem melhora estética do corpo e definição muscular.

\section{REFERÊNCIAS}

1. Sagoe D, Molde H, Andreassen CS, Torsheim T, Pallesen, S. The global epidemiology of anabolic-androgenic steroid use: a meta-analysis and meta-regression analysis. Ann Epidemiol. 2014;24(5):383-98

http://dx.doi.org/10.1016/j.annepidem.2014.01.009

2. Abrahin OSC, Sousa EC, Santos AM. Prevalence of the use of anabolic-androgenic steroids in Brazil: a systematic review. Subst Use Misuse. 2014;49(9):1156-62.

http://dx.doi.org/10.3109/10826084.2014.903750

3. Azevedo AP, Ferreira AC, Da Silva PP, Caminha IO, Freitas CM. Dismorfia muscular: a busca pelo corpo hiper musculoso. Motri. 2012;8(1):53-66.

http://dx.doi.org/10.6063/motricidade.8(1)240

4. Figueiredo VC, Silva PRP, Trindade RS, Rose EH. Doping cosmético: a problemática das aplicações intramusculares de óleos. Rev Bras Med Esporte. 2011;17(1):56-61. http://dx.doi.org/10.1590/S1517-86922011000100011
5. Darsow U, Bruckbauer H, Worret WI, Hofmann H, Ring J. Subcutaneous oleomas induced by self-injection of sesame seed oil for muscle augmentation. J Am Acad Dermatol. 2000;42(2 pt1):292-4. http://dx.doi.org/10.1016/S0190-9622(00)90144-0

6. Iversen L, Lemcke A, Bitsch M, Karlsmark T. Compression bandage as treatment for ulcers induced by intramuscular self-injection of paraffin oil. Acta Derm Venereol. 2009;89(2):196-7. http://dx.doi.org/10.2340/00015555-0583

7. Figueiredo VC, Silva PR. Cosmetic doping-when anabolic-androgenic steroids are not enough. Subst Use Misuse. 2014;49(9):1163-7. http://dx.doi.org/10.3109/10826084.2014.916522

8. Marfell-Jones M, Olds T, Stewart A, Carter L. International standards for anthropometric assessment. 2ed. Potchefstroom: International Society for the Advancement of Kinanthropometry; 2006.

9. Lukaski HC. Requirements for clinical use of bioelectrical impedance analysis (BIA). Ann NY Acad Sci. 1999;873:72-6. http://dx.doi.org/10.1111/j.1749-6632.1999.tb09451.x 
10. Pardini R, Matsudo S, Araújo T, Matsudo V, Andrade E, Braggion $\mathrm{G}$, et al. Validação do questionário internacional de atividade física (iPAQ - versão 6): estudo piloto em adultos jovens brasileiros. Rev Bras Cienc Mov. 2001;9(3):45-51.

11. Iriart JAB, Chaves JC, Orleans RG. Culto ao corpo e uso de anabolizantes entre praticantes de musculação. Cad Saúde Pública. 2009;25(4):773-82

http://dx.doi.org/10.1590/S0102-311X2009000400008

12. Iriart JAB, Andrade TM. Musculação, uso de esteróides anabolizantes e percepção de risco entre jovens fisiculturistas de um bairro popular de Salvador, Bahia, Brasil. Cad Saúde Pública. 2002;18(5):1379-87. http://dx.doi.org/10.1590/S0102-311X2002000500031
13. Schwingel PA, Cotrim HP. Consumo de recursos ergogênicos entre praticantes de musculação de academia não registrada. Lec Educ Fis Dep. 2012;17(173):e111.

14. Azevedo MPA, Ferreira ACD, Ferreira UMG. Óleos de aplicação local intramuscular: epidemiologia do uso em praticantes de musculação. Rev Bras Cienc Mov. 2009;17(3):45-53.

15. Ip EJ, Barnett MJ, Tenerowicz MJ, Perry PJ. The Anabolic 500 survey: characteristics of male users versus nonusers of anabolicandrogenic steroids for strength training. Pharmacotherapy. 2011;31(8):757-66.

http://dx.doi.org/10.1592/phco.31.8.757 